\title{
UNIQUENESS OF EMBEDDINGS OF CERTAIN INDUCED MODULES
}

\author{
J. LEPOWSKY ${ }^{1}$
}

\begin{abstract}
A theorem of D.-N. Verma on the uniqueness of embeddings of certain algebraically induced modules is generalized from the case of Borel subalgebras of semisimple Lie algebras to the case of arbitrary parabolic subalgebras.
\end{abstract}

1. Introduction. Our main purpose is to prove:

THEOREM 1.1. Let $\mathrm{g}$ be a semisimple Lie algebra over a field of characteristic zero, $\mathfrak{p}$ a parabolic subalgebra of $\mathrm{g}, L$ and $M$ one-dimensional $\mathfrak{p}$-modules, and $V^{L}$ and $V^{M}$ the corresponding (algebraically) induced $g$-modules. Then $\operatorname{dim} \operatorname{Hom}_{\mathfrak{g}}\left(V^{L}, V^{M}\right) \leqslant 1$, or equivalently, $\operatorname{dim} \operatorname{Hom}_{\mathfrak{p}}\left(L, V^{M}\right) \leqslant 1$. Moreover, every nonzero g-module map from $V^{L}$ into $V^{M}$ is an injection.

By parabolic subalgebra of $g$ we mean as usual a subalgebra whose extension to the algebraic closure of the base field contains a Borel subalgebra of the corresponding extension of $g$. Recall also that if $\mathcal{G}$ is the universal enveloping algebra of $\mathfrak{g}$ and $\mathscr{P} \subset \mathcal{G}$ the same for $\mathfrak{p}$, then the induced $\mathfrak{g}$-module $V^{L}$ is $\mathcal{G} \otimes_{\mathscr{S}} L$ (see for example $\left.[1, \S 5.1]\right)$. The equivalence of the two conditions in the theorem follows immediately from the universal property of the induced module $V^{M}$ (see for example [1, Proposition 5.1.3]). Note that Theorem 1.1 implies that $V^{L}$ can be embedded in essentially at most one way in $V^{M}$.

Theorem 1.1 generalizes D.-N. Verma's theorem [1, Théorème 7.6.6], which is the special case in which $\mathfrak{g}$ is split semisimple and $\mathfrak{p}$ is a Borel subalgebra. Our proof of the first part of Theorem 1.1 is a sharpening of Verma's proof ${ }^{2}$; the last assertion of the theorem is easy.

In a subsequent paper [3], we shall apply Theorem 1.1 to give a new proof of the uniqueness assertions concerning conical vectors in Theorems 10.1 and 10.2 of [2]; cf. the Introduction of [2].

2. Proof of Theorem 1.1. Let $k$ be the base field of characteristic zero. By extending to the algebraic closure of $k$ if necessary, we see that it is sufficient to prove Theorem 1.1 in the case in which $g$ is split semisimple with splitting

\footnotetext{
Received by the editors January 8, 1975.

AMS (MOS) subject classifications (1970). Primary 17B10; Secondary 17B35, 16A64, 22E45.

Key words and phrases. Parabolic subalgebras, algebraically induced modules, Verma modules.

${ }^{1}$ Partially supported by NSF GP 33893.

${ }^{2}$ After the work for this paper was completed, we learned that W. Borho had simplified Verma's proof. This simplification, which applies in the present generality as well, is indicated in the Remark at the end of this paper. We thank Borho for his permission to include his argument.
} 
Cartan subalgebra $\mathfrak{h}$, and $\mathfrak{p}$ contains a Borel subalgebra $\mathfrak{b}$ containing $\mathfrak{h}$. Let

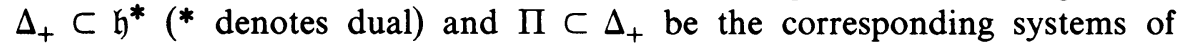
positive and simple roots, respectively. Write $\mathfrak{n}$ for the nilpotent subalgebra $\amalg \mathfrak{g}^{\varphi} \quad\left(\varphi \in \Delta_{+}\right)$of $\mathfrak{g}$ (where $\mathfrak{g}^{\varphi}$ as usual denotes the root space), so that $\mathfrak{b}=\mathfrak{h} \oplus \mathfrak{n}$. Then there is a subset $\Pi_{0}$ of $\Pi$ such that if $\Delta_{0}$ is the set of roots in the span of $\Pi_{0}$ and $\Delta_{0}^{\prime}$ the set of positive roots not in $\Delta_{0}$, then $\mathfrak{p}=\mathfrak{r}_{0} \oplus \mathfrak{n}_{0}$, where $\mathfrak{r}_{0}=\mathfrak{h} \oplus \amalg \mathfrak{g}^{\varphi}\left(\varphi \in \Delta_{0}\right)$ and $\mathfrak{n}_{0}=\amalg g^{\varphi}\left(\varphi \in \Delta_{0}^{\prime}\right)$. Also, $\mathfrak{r}_{0}$ is a reductive subalgebra of $g ; \mathfrak{n}_{0}$ is a nilpotent subalgebra; $\left[r_{0}, n_{0}\right]=n_{0} ; \Delta_{0}$ is the set of roots of $\mathfrak{r}_{0}$ with respect to its splitting Cartan subalgebra $\mathfrak{h}$; and $\Pi_{0}$ is a simple system in $\Delta_{0}$.

Clearly, $\mathfrak{r}_{0}=\mathfrak{s}_{0} \oplus \mathfrak{h}_{0}$, where $\mathfrak{s}_{0}=\left[\mathfrak{r}_{0}, \mathfrak{r}_{0}\right]$ and $\mathfrak{h}_{0}=\cap \operatorname{Ker} \varphi\left(\varphi \in \Pi_{0}\right)$. Also, $\mathfrak{s}_{0}$ is split semisimple with splitting Cartan subalgebra $\mathfrak{t}_{0}=\mathfrak{h} \cap \mathfrak{s}_{0}, \mathfrak{h}_{0}$ is the center of $\mathfrak{r}_{0}$, and $\mathfrak{h}=\mathfrak{t}_{0} \oplus \mathfrak{h}_{0}$.

Since $[\mathfrak{p}, \mathfrak{p}]=\mathfrak{s}_{0} \oplus \mathfrak{n}_{0}$, the one-dimensional $\mathfrak{p}$-modules are defined by the linear forms on $\mathfrak{p}$ which vanish on $\mathfrak{g}_{0} \oplus \mathfrak{n}_{0}$, and hence by the members of $\mathfrak{h}_{0}^{*}$. If $\lambda \in \mathfrak{h}_{0}^{*}$, extend $\lambda$ to a linear form on $\mathfrak{p}$ vanishing on $\mathfrak{S}_{0} \oplus \mathfrak{n}_{0}$; then multiplication by this linear form on $k$ defines the corresponding onedimensional $\mathfrak{p}$-module. Denote by $V^{\lambda}$ the $\mathrm{g}$-module induced by this $\mathfrak{p}$-module. Then $V^{\lambda}=\mathcal{G} \otimes_{\mathscr{S}} k$, where $\mathcal{G}$ is regarded as a right $\mathscr{P}$-module by right multiplication. What we must prove is the following:

Theorem 2.1. For all $\lambda, \mu \in \mathfrak{h}_{0}^{*}, \operatorname{dim} \operatorname{Hom}_{\mathfrak{g}}\left(V^{\lambda}, V^{\mu}\right) \leqslant 1$. Moreover, every nonzero g-module map from $V^{\lambda}$ into $V^{\mu}$ is an injection.

Let $\mathfrak{n}_{0}^{-}$be the nilpotent subalgebra $\amalg g^{\varphi}\left(\varphi \in-\Delta_{0}^{\prime}\right)$ of $\mathfrak{g}$, and $\Re_{0}^{-} \subset \mathcal{G}$ its universal enveloping algebra. Since $\mathfrak{g}=\mathfrak{n}_{0}^{-} \oplus \mathfrak{p}$, the multiplication map in $\mathcal{G}$ induces a linear isomorphism $\mathcal{G} \simeq \mathfrak{\Re}_{0} \otimes \mathscr{P}$. Let $\lambda \in \mathfrak{h}_{0}^{*}$, and let $v_{0}=1 \otimes 1$ $\in \mathcal{G} \otimes_{\mathscr{G}} k=V^{\lambda}$. Then $v_{0}$ generates $V^{\lambda}$ as a $\mathcal{G}$-module, and is called the canonical generator of $V^{\lambda}$. Clearly, the map $\omega$ : $\Re_{0}^{-} \rightarrow V^{\lambda}, x \mapsto x \cdot v_{0}$ is a linear isomorphism.

We shall use the following terminology below: If $a$ is a Lie algebra, $U$ an $a-$ module, $u \in U$ a nonzero vector and $\mu \in \mathrm{a}^{*}$, then $u$ is called a weight vector and $\mu$ a weight for $a$ if $x \cdot u=\mu(x) u$ for all $x \in$ a. For all $\mu \in \mathrm{a}^{*}$, let $U_{\mu}$ be the subspace of $U$ consisting of 0 and the weight vectors for $\mu$. Then $U_{\mu}$ is called the weight space for $\mu$. It is nonzero if and only if $\mu$ is a weight for $U$. It is clear that the canonical generator $v_{0}$ of $V^{\lambda}$ is an $\mathfrak{g}_{0} \oplus \mathfrak{n}_{0}$ - invariant weight vector for $\mathfrak{h}_{0}$ with weight $\lambda$.

Theorem 2.1 will now be proved using a series of lemmas. The first of these proves the second part of the theorem.

Lemma 2.2. Let $\lambda \in \mathfrak{h}_{0}^{*}$ and $v \in V^{\lambda}, v \neq 0$. Then the map from $\Re_{0}^{-}$into $V^{\lambda}$ taking $x \in \Re_{0}^{-}$to $x \cdot v$ is injective. Moreover, if $\mu \in \mathfrak{h}_{0}^{*}$, then any nonzero $\mathrm{g}$ module map from $V^{\mu}$ into $V^{\lambda}$ is injective.

Proof. Since the map $\omega$ (see above) is a linear isomorphism, we may write $v$ as $y \cdot v_{0}$, where $y \in \Re_{0}^{-}, y \neq 0$. If $x \in \Re_{0}^{-}, x \neq 0$, then $x y \neq 0$ in $\Re_{0}^{-}$ since $\Re_{0}^{-}$has no zero divisors. Thus $x y \cdot v_{0} \neq 0$ in $V^{\lambda}$, so that $x \cdot v \neq 0$. This proves the first assertion. To prove the second, let $v_{0}^{\prime}$ be the canonical generator of $V^{\mu}$ and let $f: V^{\mu} \rightarrow V^{\lambda}$ be a nonzero g-module map. Then $v=f\left(v_{0}^{\prime}\right) \neq 0$. Let $w \in V^{\mu}, w \neq 0$. Then $w=z \cdot v_{0}^{\prime}$ for some $z \in \Re_{0}^{-}, z$ $\neq 0$. Hence $f(w)=z \cdot v \neq 0$ by the first assertion of the lemma. Q.E.D. 
LEMmA 2.3. Let $\lambda \in \mathfrak{h}_{0}^{*}$. Then $V^{\lambda}$ contains an irreducible $\mathfrak{g}$-submodule generated by an $\mathfrak{n}$-invariant weight vector for $\mathfrak{h}$.

Proof. Since $V^{\lambda}$ is generated by an $n$-invariant weight vector for $\mathfrak{h}$, the canonical generator, $V^{\lambda}$ is the g-module quotient of a Verma module $V$ (i.e., a $g$-module induced by a one-dimensional b-module) by a submodule $W$ of $V$ (see [1, Proposition 7.1.8(i)]). By the standard properties of Verma modules (see [1, Proposition 7.6.1]), $V$ has a composition series, and every irreducible subquotient of $V$ is generated by an $\mathfrak{n}$-invariant weight vector for $\mathfrak{h}$. Thus the same is true of $V / W$, proving the lemma. Q.E.D.

Let $\psi_{1}, \ldots, \psi_{r} \in \mathfrak{h}_{0}^{*}$ be the (not necessarily distinct) restrictions to $\mathfrak{h}_{0}$ of the roots in $\Delta_{0}^{\prime}$. Note that each $\psi_{i} \neq 0$. For all $\psi \in \mathfrak{h}_{0}^{*}$, define $P_{0}(\psi) \in \mathbf{Z}_{+}$to be the number of sequences $n_{1}, \ldots, n_{r}$ of nonnegative integers such that $\psi$ $=\sum_{i=1}^{r} n_{i} \psi_{i}$. The next two results relate the "partition function" $P_{0}$ to multiplicities of weights.

LEMma 2.4. Under the adjoint action of $\mathfrak{h}_{0}$ on $\Re_{0}^{-}, \Re_{0}^{-}$is a direct sum of its weight spaces for $\mathfrak{h}_{0}$. For all $\mu \in \mathfrak{h}_{0}^{*}, \mu$ is a weight for $\mathfrak{\Re}_{0}^{-}$if and only if $P_{0}(-\mu)>0$, and $\operatorname{dim}\left(\varkappa_{0}^{-}\right)_{\mu}=P_{0}(-\mu)$.

Proof. Let $\varphi_{1}, \ldots, \varphi_{r}$ be the elements of $\Delta_{0}^{\prime}$, labeled so that their restrictions to $\mathfrak{h}_{0}$ are $\psi_{1}, \ldots, \psi_{r}$, respectively. Let $e_{1}, \ldots, e_{r}$ be nonzero elements in $\mathrm{g}^{-\varphi_{1}}, \ldots, \mathrm{g}^{-\varphi_{r}}$, respectively. Then $e_{1}, \ldots, e_{r}$ form a basis of $\mathrm{n}_{0}^{-}$, and so the monomials $e_{1}^{n_{1}} e_{2}^{n_{2}} \cdots e_{r}^{n_{r}}$ form a basis of $\Re_{0}^{-}$, as $\left\{n_{1}, \ldots, n_{r}\right\}$ varies among the sequences of nonnegative integers. Since the indicated monomial is a weight vector with weight $-\sum_{i=1}^{r} n_{i} \psi_{i}$ for $\mathfrak{h}_{0}$, the lemma is proved. Q.E.D.

Lemma 2.5. For all $\lambda \in \mathfrak{h}_{0}^{*}, V^{\lambda}$ is a direct sum of its weight spaces for $\mathfrak{h}_{0}$. For all $\mu \in \mathfrak{h}_{0}^{*}, \operatorname{dim} V_{\mu}^{\lambda}=P_{0}(\lambda-\mu)$. More generally, if $\nu \in \mathfrak{h}_{0}^{*}$ and $v \in V_{\nu}^{\lambda}, v$ $\neq 0$, then $\operatorname{dim}\left(\Re_{0}^{-} \cdot v\right)_{\mu}=P_{0}(\nu-\mu)$.

Proof. This result follows from Lemma 2.4 and the first part of Lemma 2.2, together with the fact that for all $\mu \in \mathfrak{h}_{0}^{*},\left(\Re_{0}^{-}\right)_{\mu-\nu} \cdot v$ is included in and hence equals $\left(\pi_{0}^{-} \cdot v\right)_{\mu}$. Q.E.D.

Lemma 2.6. Let $\lambda, \mu \in \mathfrak{h}_{0}^{*}$, and suppose that $\operatorname{dim} \operatorname{Hom}_{\mathfrak{g}}\left(V^{\lambda}, V^{\mu}\right)>1$. Then there exists $\nu \in \mathfrak{h}_{0}^{*}$ such that for all $\sigma \in \mathfrak{h}_{0}^{*}$ and $n \in \mathbf{Z}_{+}, P_{0}(\sigma+n(\mu-\nu))$ $\geqslant 2^{n} P_{0}(\sigma)$.

Proof. Let $V$ be an irreducible g-module isomorphic to a g-submodule of $V^{\lambda}$ and generated by an $\mathfrak{n}$-invariant weight vector for $\mathfrak{h}$ (see Lemma 2.3), and choose any $\nu \in \mathfrak{h}_{0}^{*}$ such that $V_{\nu} \neq 0$, and any $v \in V_{v}, v \neq 0$. Composition with the injection from $V$ into $V^{\lambda}$ gives a natural map $\operatorname{Hom}_{\mathfrak{a}}\left(V^{\lambda}, V^{\mu}\right)$ $\rightarrow \operatorname{Hom}_{\mathfrak{g}}\left(V, V^{\mu}\right)$. By the second part of Lemma 2.2, this map is injective, and so $\operatorname{dim} \operatorname{Hom}_{\mathfrak{g}}\left(V, V^{\mu}\right)>1$. Let $f$ and $g$ be two linearly independent $\mathrm{g}$-maps from $V$ into $V^{\mu}$. Then $f(V) \neq g(V)$, because $f(V)=g(V)$ would imply that $V$ admits a nonscalar g-module automorphism, a contradiction (see [1, Proposition 7.1.8(iv)]). Since $V$ is irreducible, $f(V) \cap g(V)=0$. Hence $\Re_{0}^{-} \cdot f(v) \cap \Re_{0}^{-} \cdot g(v)=0$, and so for all $\tau \in \mathfrak{h}_{0}^{*}$,

$$
P_{0}(\mu-\tau)=\operatorname{dim} V_{\tau}^{\mu} \geqslant \operatorname{dim}\left(\Re_{0}^{-} \cdot f(\nu)\right)_{\tau}+\operatorname{dim}\left(\Re_{0}^{-} \cdot g(v)\right)_{\tau}=2 P_{0}(\nu-\tau),
$$

by Lemma 2.5. Setting $\sigma=\nu-\tau$, we get $P_{0}(\sigma+(\mu-\nu)) \geqslant 2 P_{0}(\sigma)$ for all 
$\sigma \in \mathfrak{h}_{0}^{*}$, and the lemma is now immediate. Q.E.D.

LemMa 2.7. The restrictions $\alpha_{1}, \ldots, \alpha_{p}$ to $\mathfrak{h}_{0}^{*}$ of the members of $\Pi-\Pi_{0}$ form a basis of $\mathfrak{h}_{0}^{*}$. For all $\xi \in \mathfrak{h}_{0}^{*}$ such that $\xi$ is a nonnegative integral linear combination of the $\alpha_{i}$ 's, let $s(\xi)$ be the sum of the coefficients in the corresponding expansion of $\xi$. Then $P_{0}(\xi) \leqslant(s(\xi)+1)^{r}$, where $r$ is, as above, the cardinality of $\Delta_{0}^{\prime}$.

Proof. The first assertion is clear. To prove the second, we just imitate the argument in [1, Lemme 7.6.5]: Recall that $\psi_{1}, \ldots, \psi_{r}$ are the (not necessarily distinct) restrictions to $\mathfrak{h}_{0}$ of the roots in $\Delta_{0}^{\prime}$, and note that these are nonnegative integral linear combinations of the $\alpha_{i}$ 's. For all $\xi$ as in the statement of the lemma and $j=1, \ldots, r$, define $P_{j}(\xi) \in \mathbf{Z}_{+}$to be the number of sequences $n_{1}, \ldots, n_{j}$ of nonnegative integers such that $\xi=\sum_{i=1}^{j} n_{i} \psi_{i}$. Note that $P_{r}(\xi)$ is the original partition function $P_{0}(\xi)$. We show by induction on $j$ that $P_{j}(\xi) \leqslant(s(\xi)+1)^{j}$. This is clearly true if $j=1$. Suppose it is true for $j$. In the following expressions, the sums are over all $\xi-n \psi_{j+1}\left(n \in \mathbf{Z}_{+}\right)$ which are nonnegative integral linear combinations of the $\alpha_{i}$ 's. We have

$$
\begin{aligned}
P_{j+1}(\xi) & =\sum P_{j}\left(\xi-n \psi_{j+1}\right) \leqslant \sum\left(s\left(\xi-n \psi_{j+1}\right)+1\right)^{j} \\
& \leqslant(s(\xi)+1)(s(\xi)+1)^{j}=(s(\xi)+1)^{j+1},
\end{aligned}
$$

proving the desired formula. Q.E.D.

It is now easy to complete the proof of Theorem 2.1. Suppose $\lambda, \mu \in \mathfrak{h}_{0}^{*}$ and $\operatorname{dim} \operatorname{Hom}_{\mathfrak{g}}\left(V^{\lambda}, V^{\mu}\right)>1$. Taking $\sigma=0$ in Lemma 2.6, we get $P_{0}(n(\mu-\nu))$ $\geqslant 2^{n}$ for all $n \in \mathbf{Z}_{+}$. Since $2^{n}>0, n(\mu-\nu)$ is a nonnegative integral linear combination of $\alpha_{1}, \ldots, \alpha_{p}$. Hence by Lemma $2.7,(n s(\mu-\nu)+1)^{r} \geqslant 2^{n}$ for all $n \in \mathbf{Z}_{+}$, an impossibility. This proves Theorem 2.1 and hence also Theorem 1.1. Q.E.D.

REMARK. Here is Borho's simplification of our argument (see footnote 2): After Lemma 2.3, we may omit everything (including the partition function) except the proof of Lemma 2.6 through the statement " $\Re_{0}^{-} \cdot f(v) \cap \Re_{0}^{-} \cdot g(v)$ $=0$ ", after which we add the following paragraph:

Writing $f(v)=n_{1} \cdot v_{0}$ and $g(v)=n_{2} \cdot v_{0}$, where $v_{0}$ is the canonical generator of $V^{\mu}$ and $n_{1}, n_{2} \in \Re_{0}^{-}$, we see that the algebra $\Re_{0}^{-}$contains two disjoint nonzero principal left ideals $\pi_{0}^{-} n_{1}$ and $\varkappa_{0}^{-} n_{2}$. But this contradicts Goldie's result that any two nonzero elements of the universal enveloping algebra of a finite-dimensional Lie algebra have a common nonzero left multiple. (The simple Lesieur-Croisot proof of this "Ore condition" is given in $[4, \mathrm{pp}$. 165-166].) This proves Theorem 2.1.

\section{REFERENCES}

1. J. Dixmier, Algèbres enveloppantes, Gauthier-Villars, Paris, 1974.

2. J. Lepowsky, Conical vectors in induced modules, Trans. Amer. Math. Soc. 208 (1975), 219-272.

3. - On the uniqueness of conical vectors, Proc. Amer. Math. Soc. (to appear).

4. N. Jacobson, Lie algebras, Interscience Tracts in Pure and Appl. Math., no. 10, Interscience, New York, 1962. MR 26 \# 1345. 